\title{
Band-structure-based collisional model for electronic excitations in ion-surface collisions
}

\author{
M. N. Faraggi, ${ }^{1}$ M. S. Gravielle, ${ }^{1,2}$ M. Alducin, ${ }^{3}$ J. I. Juaristi, ${ }^{4,5}$ and V. M. Silkin $^{3}$ \\ ${ }^{1}$ Instituto de Astronomía y Física del Espacio, CONICET, Casilla de Correo 67, Sucursal 28, 1428 Buenos Aires, Argentina \\ ${ }^{2}$ Departamento de Física, FCEN, Universidad de Buenos Aires, Buenos Aires, Argentina \\ ${ }^{3}$ Donostia International Physics Center DIPC, P. Manuel de Lardizabal 4, 20018 San Sebastián, Spain \\ ${ }^{4}$ Departamento de Física de Materiales Facultad de Químicas, UPV/EHU, Apartado 1072, 20080 San Sebastián, Spain \\ ${ }^{5}$ Unidad de Física de Materiales Centro Mixto CSIC-UPV/EHU, Facultad de Químicas, \\ UPV/EHU, Apartado 1072, 20080 San Sebastián, Spain
}

(Received 14 April 2005; published 15 July 2005)

\begin{abstract}
Energy loss per unit path in grazing collisions with metal surfaces is studied by using the collisional and dielectric formalisms. Within both theories we make use of the band-structure-based (BSB) model to represent the surface interaction. The BSB approach is based on a model potential and provides a precise description of the one-electron states and the surface-induced potential. The method is applied to evaluate the energy lost by $100 \mathrm{keV}$ protons impinging on aluminum surfaces at glancing angles. We found that when the realistic BSB description of the surface is used, the energy loss obtained from the collisional formalism agrees with the dielectric one, which includes not only binary but also plasmon excitations. The distance-dependent stopping power derived from the BSB model is in good agreement with available experimental data. We have also investigated the influence of the surface band structure in collisions with the $\mathrm{Al}(100)$ surface. Surface-state contributions to the energy loss and electron emission probability are analyzed.
\end{abstract}

DOI: 10.1103/PhysRevA.72.012901

PACS number(s): 34.50.Dy, 34.50.Bw

\section{INTRODUCTION}

When an energetic ion collides with a metal surface with a glancing angle, it excites conduction electrons of the solid [1]. The energy lost by the projectile in this process can be calculated using two different approaches: the dielectric and collisional formalisms. Results obtained with both theories depend on the model employed to describe the surface interaction. For usual surface potentials the dielectric approach includes both the binary and collective mechanisms of energy loss [2-7]. While the former mechanism, also named electron-hole creation, takes into account single collisions of the projectile with valence electrons, the latter involves the collective response of the medium to the incident ion, i.e., the excitation of the plasmon field.

The collisional theory, instead, is useful to derive angle and energy distributions of electrons ejected as a result of the collision [8], which are not easily accessible from the dielectric formalism. But it failed to calculate the energy lost by the projectile when different approximated models were used to represent the surface interaction [9]. The failure of these collisional results was attributed to the fact that the component of the transferred momentum perpendicular to the surface was absent or included in an average way in the wake potential. To solve this problem, in a previous work [10] we proposed an approximate model for the induced potential that corrects the deficiency, but without including plasmon excitations.

The purpose of this work is to investigate the performance of the collisional formalism to evaluate the energy loss when an accurate description of the surface potential is considered. To represent the electron-surface interaction we employ the recently proposed band-structure-based (BSB) model [11], which describes the potential at the surface as a finite and smooth barrier. The BSB model incorporates information about the band structure of the solid [12] and has been successfully applied in several branches [13-16]. It provides not only the surface-induced potential but also the unperturbed electronic states, both calculated in a consistent way.

Our study is focused on $100 \mathrm{keV}$ protons impinging at grazing angles on aluminum surfaces. For $\mathrm{Al}(111)$ we analyze the energy loss derived from the collisional formalism by comparing the results with those obtained from the dielectric approach. Both theories are evaluated with the same surface interaction, given by the BSB model. Unlike previous findings, where simpler surface descriptions were considered [9], we have found that collisional and dielectric distancedependent stopping powers coincide quite well for different projectile-surface distances. In addition, we investigated the importance of the surface band structure by considering the $\mathrm{Al}(100)$ surface. For this crystal orientation the presence of an occupied surface state is expected to introduce changes in the energy loss and electron emission spectra.

The work is organized as follows. In Sec. II we describe the theoretical models, in Sec. III, results are presented and discussed, and Sec. IV contains the conclusions. Atomic units are used unless otherwise stated $\left(e^{2}=\hbar=m_{e}=1\right)$.

\section{THEORETICAL MODEL}

We consider a heavy projectile $(P)$ of charge $Z_{P}$ and mass $M_{P}$ impinging at grazing angles with velocity $\mathbf{v}$ on a solid surface $(S)$. As a result of the collision, an electron $(e)$ of the conduction band of the solid, initially in the state $i$, is excited to a final state $f$. The frame of reference is fixed to the position of the topmost atomic layer, with the $\hat{z}$ axis perpendicular to the surface, aiming toward the vacuum region.

For glancing impact angles, the classical path of the ion can be divided into differential portions parallel to the sur- 
face at different distances $Z$. In every portion, the component of the ion velocity perpendicular to the surface can be neglected and the projectile position at a given time $t$ reads $\mathbf{R}(t)=\left(\mathbf{R}_{s}(t), Z\right)$, where $\mathbf{R}_{s}(t)=\mathbf{v} t$ denotes the coordinates parallel to the surface plane.

For the considered one-active-electron system, the electronic Hamiltonian reads

$$
H(t)=H_{0}+V_{P e}(t),
$$

where $H_{0}=-1 / 2 \nabla_{\mathbf{r}}^{2}+V_{S e}$ represents the unperturbed Hamiltonian, and $V_{S e}$ and $V_{P e}(t)$ are the interactions of the electron with the solid surface and the projectile, respectively. The potential $V_{P e}(t)$ includes the shielding of the projectile interaction produced by the presence of the other valence electrons, being

$$
V_{P e}(\mathbf{r}, t)=-Z_{P} /|\mathbf{r}-\mathbf{R}(t)|+V_{\text {ind }}(\mathbf{r}, t),
$$

where $V_{\text {ind }}(\mathbf{r}, t)$ is the surface potential induced by the incident ion.

We use the BSB model to describe the surface interactions $V_{S e}$ and $V_{\text {ind }}$. In the BSB model, translational invariance in the plane parallel to the surface is assumed and the eigenfunctions of $H_{0}$ are expressed as

$$
\Phi_{\mathbf{k}_{s}, n}(\mathbf{r})=\frac{1}{2 \pi} \exp \left(i \mathbf{r}_{s} \cdot \mathbf{k}_{s}\right) \phi_{n}(z),
$$

where $\mathbf{r}=\left(\mathbf{r}_{s}, z\right)$ is the electron position vector, $\mathbf{k}_{s}$ is the electron momentum parallel to the surface, and $E=k_{s}^{2} / 2+\varepsilon_{n}$ is the eigenenergy. The one-dimensional functions $\phi_{n}(z)$ and their corresponding eigenenergies $\varepsilon_{n}$ are obtained by solving the one-electron Schrödinger equation associated with the realistic one-dimensional model potential of Ref. [11]. This potential describes in an appropriate way the main characteristics of the surface band structure such as the surface band gap and the surface state. In the resolution of the onedimensional Schrödinger equation we employ a slab geometry with the following representation for $\phi_{n}(z)$ :

$$
\phi_{n}(z)=\frac{1}{\sqrt{L}} \sum_{j=-N}^{N} a_{n}(j) \exp \left(i \frac{2 \pi j}{L} \widetilde{z}\right),
$$

where $L$ is a normalization length and $2 N+1$ is the number of basis functions. The coordinate $\tilde{z}=z+d_{S}$ is measured with respect to the center of the slab, which is placed at a distance $d_{S}$ from the surface atomic plane, and the coefficients $a_{n}(j)$ are numerically evaluated.

The BSB induced potential is obtained in linear response theory by employing the BSB electronic states, given by Eq. (3). It is expressed as

$$
V_{\text {ind }}(\mathbf{r}, t)=Z_{P} \int \frac{d^{2} \mathbf{q}_{s}}{(2 \pi)^{2}} e^{i \mathbf{q}_{s} \cdot\left(\mathbf{r}_{s}-\mathbf{v} t\right)} W_{\text {ind }}\left(q_{s}, z, Z ; \omega\right),
$$

where $W_{\text {ind }}\left(q_{s}, z, Z ; \omega\right)$ is the Fourier transform respect to $\mathbf{r}_{s}$ and $t$ of the induced interaction obtained within the randomphase approximation, and $\omega=\mathbf{q}_{s} \cdot \mathbf{v}$.

In this work we are interested in evaluating the energy loss per unit path length, $\mathcal{S}(Z)$, when the ion moves parallel to the surface at a given distance $Z$. This magnitude, also called distance-dependent stopping power, will be calculated by employing the BSB model within two different approaches: the collisional and the dielectric approximations.

\section{A. Collisional BSB approximation}

Within the collisional formalism, when the projectile moves at a distance $Z$ from the surface, the transition probability per unit path reads

$$
\mathcal{P}_{i f}(Z)=\frac{2 \pi}{v} \rho_{e} \delta(\Delta)\left|T_{i f}\right|^{2},
$$

where $T_{i f}$ denotes the $T$-matrix element for the inelastic transition $\Phi_{\mathbf{k}_{i s}, n_{i}} \rightarrow \Phi_{\mathbf{k}_{f s}, n_{f}}, \rho_{e}=2$ takes into account the spin states, and $v=|\mathbf{v}|$. In Eq. (6), the $\delta$ function imposes the energy conservation $\Delta=\mathbf{v} \cdot\left(\mathbf{k}_{f s}-\mathbf{k}_{i s}\right)-E_{i f}$, where $\mathbf{k}_{i s}\left(\mathbf{k}_{f s}\right)$ is the initial (final) electron momentum parallel to the surface and $E_{i f}=E_{f}-E_{i}$ is the energy gained by the electron or lost by the projectile in the transition, with $E_{i}\left(E_{f}\right)$ being the initial (final) electron energy.

Using the BSB model to represent the surface interaction, the transition matrix within first-order perturbation theory [17] reads

$$
\begin{aligned}
T_{i f}^{(B S B)}= & \frac{-Z_{P}}{(2 \pi)^{3} L} \sum_{j=-N}^{N} a_{n_{f}}^{*}(j) \sum_{j^{\prime}=-N}^{N} a_{n_{i}}\left(j^{\prime}\right) \\
& \times\left\{\frac{2(2 \pi)^{2} \exp \left[-i Q_{j j^{\prime}}\left(Z+d_{S}\right)\right]}{q_{S}^{2}+Q_{j j^{\prime}}^{2}}\right. \\
& \left.+\tilde{W}_{i n d}\left(q_{s}, Q_{j j^{\prime}}, Z ; \omega\right)\right\},
\end{aligned}
$$

where $\tilde{W}_{\text {ind }}\left(q_{s}, q_{z}, Z ; \omega\right)$ is the Fourier transform with respect to $z$ of the screening function $W_{\text {ind }}\left(q_{s}, z, Z ; \omega\right), \mathbf{q}_{s}=\mathbf{k}_{f s}-\mathbf{k}_{i s}$ is the electronic momentum transfer parallel to the surface, and $Q_{j j^{\prime}}=2 \pi\left(j-j^{\prime}\right) / L$.

The collisional distance-dependent stopping power is derived from Eq. (6) by multiplying $\mathcal{P}_{\text {if }}$ by the lost energy and adding over all possible initial and final states:

$$
\begin{aligned}
\mathcal{S}_{c o l}^{(B S B)}(Z)= & \sum_{n_{f}} \sum_{n_{i}} \int d \mathbf{k}_{f s} \int d \mathbf{k}_{i s} \Theta\left(-E_{W}-E_{i}\right) \\
& \times \Theta\left(E_{f}+E_{W}\right) E_{i f} \mathcal{P}_{i f}(Z),
\end{aligned}
$$

where $\Theta$ is the unitary Heaviside function and $E_{W}$ is the work function. The function $\Theta\left(-E_{W}-E_{i}\right)$ has been introduced in Eq. (8) to restrict the initial states to those contained inside the Fermi sphere, while $\Theta\left(E_{f}+E_{W}\right)$ takes into account the Pauli exclusion principle.

\section{B. Dielectric BSB approximation}

Within the dielectric formalism, the energy lost by the projectile per unit path is expressed in terms of the retarding force produced by the induced potential $V_{i n d}$, which also acts on the projectile. Then, it reads 


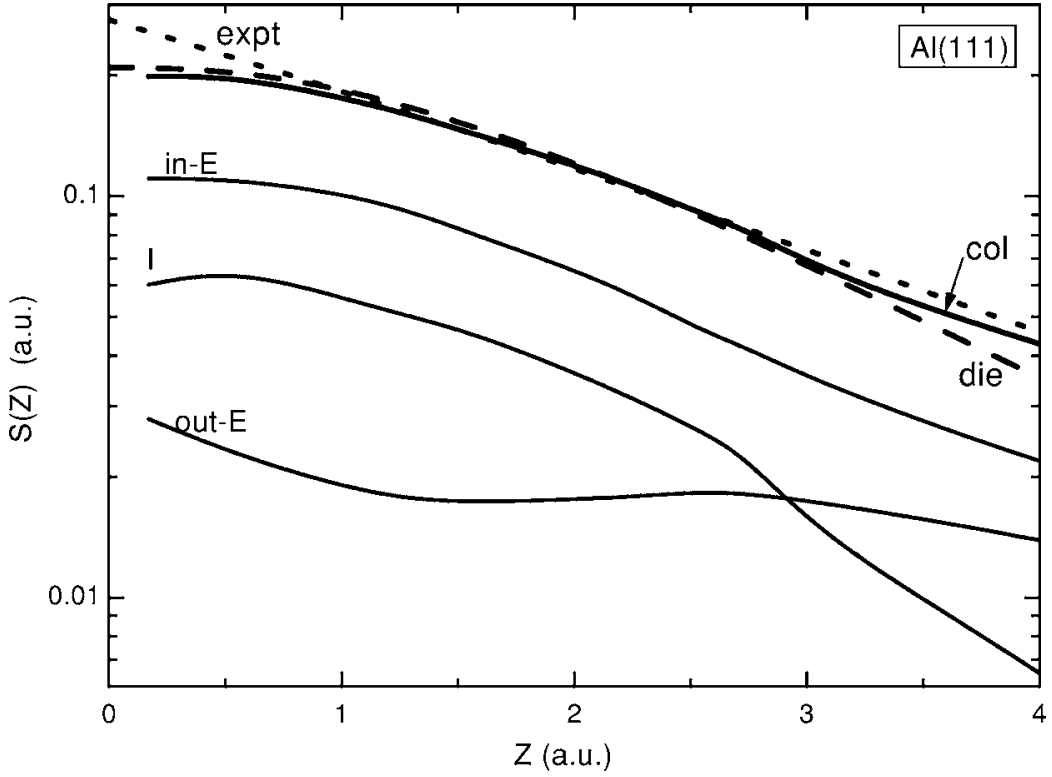

FIG. 1. Energy loss per unit path, $\mathcal{S}(Z)$, as a function of the projectile distance to the topmost atomic layer, for $100 \mathrm{keV}$ protons impinging on $\mathrm{Al}(111)$ surfaces. Thick solid and dashed lines, collisional and dielectric BSB approximations, respectively; dotted line, experimentally derived data for $120 \mathrm{keV}$ proton impact, extracted from Ref. [18]. Thin solid lines, partial contributions from in-E, out-E, and I processes, as explained in the text.

$$
\mathcal{S}_{\text {die }}(Z)=-\frac{1}{v} \int d \mathbf{r}^{\prime} \rho_{\text {ext }}\left(\mathbf{r}^{\prime}, t\right) \frac{\partial V_{\text {ind }}\left(\mathbf{r}^{\prime}, t\right)}{\partial t},
$$

where $\rho_{\text {ext }}\left(\mathbf{r}^{\prime}, t\right)$ represents the incident-particle charge density. In our case, $\rho_{\text {ext }}$ corresponds to a point charge $Z_{P}$ moving parallel to the surface with velocity $\mathbf{v}$ at a distance $Z$ from the surface, i.e.,

$$
\rho_{\text {ext }}\left(\mathbf{r}^{\prime}, t\right)=Z_{P} \delta\left(\mathbf{r}^{\prime}{ }_{s}-\mathbf{v} t\right) \delta\left(z^{\prime}-Z\right),
$$

with $\mathbf{r}^{\prime}=\left(\mathbf{r}^{\prime}{ }_{s}, z^{\prime}\right)$. Employing the induced potential derived from the BSB model [Eq. (5)] and making use of the parity properties of $W_{\text {ind }}$, the dielectric distance-dependent stopping power reads [3]

$$
\begin{aligned}
\mathcal{S}_{\text {die }}^{(B S B)}(Z)= & -\frac{2 Z_{P}^{2}}{v} \int \frac{d \mathbf{q}_{s}}{(2 \pi)^{2}} \mathbf{q}_{s} \cdot \mathbf{v} \Theta\left(\mathbf{q}_{s} \cdot \mathbf{v}\right) \\
& \times \operatorname{Im}\left[W_{\text {ind }}\left(q_{s}, Z, Z ; \mathbf{q}_{s} \cdot \mathbf{v}\right)\right] .
\end{aligned}
$$

\section{RESULTS}

The collisional system composed of $100 \mathrm{keV}$ protons impinging on an aluminum surface with a glancing incidence angle is here employed as a benchmark for the theory. For this particular system, experimental data have been reported in Ref. [18]. Here, we show results for two different crystal surfaces: $\mathrm{Al}(111)$ and $\mathrm{Al}(100)$. We use the Fermi energy $E_{F}$ $=11.27 \mathrm{eV}$, the work functions $E_{W}=4.24$ and $4.4 \mathrm{eV}$, and the interplanar distances 4.388 and 3.80 a.u. for the (111) and (100) crystal orientations, respectively [11].

The one-dimensional Schrödinger equation corresponding to the BSB model is solved with the plane-wave basis set of Eq. (4). The parameters used to describe the $\mathrm{Al}(111)[\mathrm{Al}(100)]$ surface are $N=170$ (220), $L$ $=394.92$ (342.038) a.u., and $d_{S}=155.77$ (134.9) a.u.

Within the collisional formalism, the evaluation of the energy loss per unit path involves a sum of four-dimensional integrals [see Eq. (8)]. We solve analytically one of these integrals by means of the $\delta$ function contained in Eq. (6), whereas the others are calculated by employing the Monte Carlo numerical technique with a relative error of less than $3 \%$. To reduce computing time, the sum over the quantum number $n_{f}$ contained in $\mathcal{S}_{c o l}^{(B S B)}(Z)$ is evaluated by interpolating values for the highest $n_{f}$, where the terms of the sum smoothly decrease. As the use of a discrete basis set imposes a limit in the minimum momentum transfer, we have introduced a cutoff $k_{\varepsilon} \simeq 0.1$ a.u. around the minimum value of $k_{f s}$ for electronic transitions with $\varepsilon_{n_{f}}<-E_{W}$.

Within the dielectric approach, the distance-dependent stopping power is obtained from Eq. (11). In this case, the integrals are calculated numerically with a relative error of $\sim 1 \%$.

\section{A. Energy loss}

We start the study by considering the (111) crystal orientation. In Fig. 1 we plot the distance-dependent stopping power for the $\mathrm{Al}(111)$ surface, as a function of the projectile distance to the topmost atomic layer. Collisional and dielectric BSB results of the energy loss are displayed in the figure, and both curves run very close to each other for the different $Z$ values. This agreement can be considered as an indication of the good behavior of the collisional BSB theory. The collisional approach strongly depends on the model used to represent the surface potential. It requires a precise description of the momentum transfer perpendicular to the surface, and when the usual induced potentials were used, questionable values of the energy loss-larger than dielectric ones-were found [9]. The electronic momentum transferred in the collision, $\mathbf{Q}=\left(\mathbf{q}_{s}, Q_{j j^{\prime}}\right)$, is contained in $\mathcal{S}_{c o l}^{(B S B)}(Z)$ through the Fourier transform of $V_{P e}$, as given by Eq. (7). Since in the BSB model, $\tilde{W}_{\text {ind }}$ properly represents the distribution of the perpendicular momentum $Q_{j j^{\prime}}$, it leads to reliable collisional results for the energy loss. These collisional values are similar to those derived from the dielectric formalism, which includes binary plus collective excitations. The separation 
between the collisional and dielectric BSB curves for $Z$ $\geq 3.5$ a.u. might be attributed to a poor description of the low-transferred-momentum region, due to the use of a discrete basis. In addition, as can be seen from Fig. 1, collisional BSB values display a very good agreement with experimentally derived data for a slightly different impact energy, which were extracted from Ref. [18]. The underestimation of the experimental values for $Z \leqq 0.5$ a.u. can be attributed to inner-shell excitations, which are relevant for small distances to the topmost atomic layer [4].

The collisional approach allows us to separate easily the contributions to the energy loss coming from the different electronic processes. In this work, we have classified the final collision channels according to the $\varepsilon_{n_{f}}$ values, taking into account that final states with total energy $E_{f}=k_{f s}^{2} / 2+\varepsilon_{n_{f}}$ lower than $-E_{W}$ are initially occupied and transitions to such states are forbidden. Allowed electronic transitions were grouped as inner excitation (in-E), outer excitation (out-E), and ionization (I) processes. The excitation processes correspond to transitions to final states bound to the surface, with $\varepsilon_{n_{f}}<0, \varepsilon_{n_{f}}$ being lower (higher) than $-E_{W}$ for the in-E (out-E) mechanism. The I process, instead, corresponds to electrons ejected outside or inside the solid, and it is associated with final electronic states with $\varepsilon_{n_{f}}>0$. In Fig. 1 we also show the collisional energy losses corresponding to the different electronic processes as a function of the $Z$ distance. The in-E mechanism dominates in the whole range of considered $Z$ distances, contributing $40 \%$ more than the I process. At small distances from the surface, the out-E energy loss is lower than the I contribution, but it increases as $Z$ augments, dominating over the I process for large $Z$ values.

In a detailed examination of the proposed approximation, we analyze the differential distribution of energy loss $d \mathcal{S} / d E_{i f}$, which is derived from Eq. (8) taking into consideration that $\mathcal{S}(Z)$ can be also expressed as $\mathcal{S}(Z)$ $=\int d E_{i f}\left(d \mathcal{S} / d E_{i f}\right)$. Collisional and dielectric BSB values of $d \mathcal{S} / d E_{i f}$ are shown in Fig. 2 as a function of the lost energy. We consider the projectile distance $Z=4.2$ a.u., for which the surface plasmon contribution is expected to be important. The dielectric BSB distribution displays a pronounced peak at the surface plasmon frequency $\omega_{s}$ (shifted due to the plasmon dispersion), which is associated with the collective absorption of energy due to plasmon excitations. But this peak is not present in the collisional BSB curve, which shows a smoothly decreasing behavior. While for high values of $E_{i f}$ both theories agree, indicating that they are describing head-on Coulomb collisions, at low energies the collisional approach gives a larger contribution than the dielectric one. The reason behind this effect is that the surface plasmon has a finite width and is coupled to the electron-hole continuum. As a consequence of this coupling, in the dielectric formalism that describes the energy deposition in a many-body system of interacting particles, there is a reduction of the strength for low-energy single-particle excitations. However, in the collisional formalism, where-by construction-the transferred energy is finally absorbed by a single independent electron, the weight of the collective and many-particle excitations is distributed among low-energy single-particle excitations [19]. This may be seen as if the collisional model

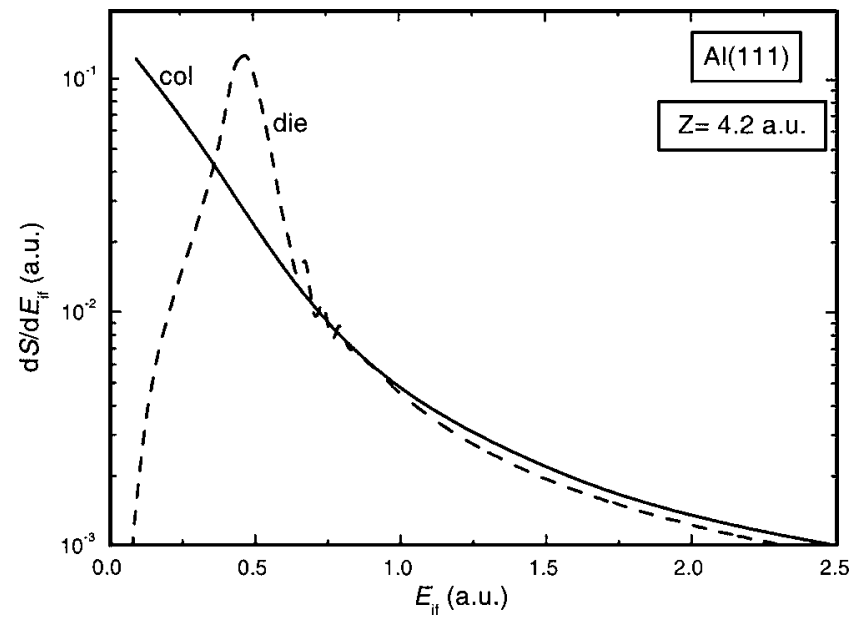

FIG. 2. Differential distribution of energy loss per unit path, $d \mathcal{S} / d E_{i f}$, as a function of the transferred energy, for $\mathrm{Al}(111)$ surfaces. The projectile distance is $Z=4.2$ a.u., measured with respect to the topmost atomic layer. Solid and dashed lines, collisional and dielectric BSB approximations, respectively.

averaged contributions from collective excitations. The increased probability for low-energy excitations in the collisional formalism compensates the lack of plasmon excitations. As a result, the stopping power values given by the two models are very similar (Fig. 1).

\section{B. Band structure effect}

With the aim of studying the influence of the surface crystal structure we have considered the $\mathrm{Al}(100)$ surface. This crystal surface presents an occupied surface state located at the energy $-7.15 \mathrm{eV}$ measured from the vacuum level, whose contribution is estimated to be more important than in the case of $\mathrm{Al}(111)$, as a consequence of its proximity to the Fermi level. Since the BSB model incorporates information about the band structure of the solid, the presence of surface states should produce some effect on the BSB energy loss. In Fig. 3(a) we plot the distance-dependent stopping power for the $\mathrm{Al}(100)$ surface, as a function of the projectile distance to the topmost atomic layer. Again, collisional and dielectric approximations are in good agreement, indicating the reliability of the collisional BSB method. Note that at the considered impact energy, BSB results for the two crystal orientations-(111) and (100) — are very similar; they differ by only about $5 \%$. Collisional BSB values derived without including the surface state are also shown in Fig. 3(a). We observe that when the surface state is excluded from the calculation, the collisional BSB theory underestimates the value of the energy loss. The contribution of the surface state increases for large $Z$ values, where the excitation probability from internal bound states is lower. In Fig. 3(b) one can see that the presence of the surface state essentially affects the excitation processes (in-E and out-E mechanisms) while for the ionization process its contribution is smaller.

For $\mathrm{Al}(100)$ we also analyze differential electron emission spectra, which are expected to reveal detailed information about the crystal surface. The differential probability of va- 


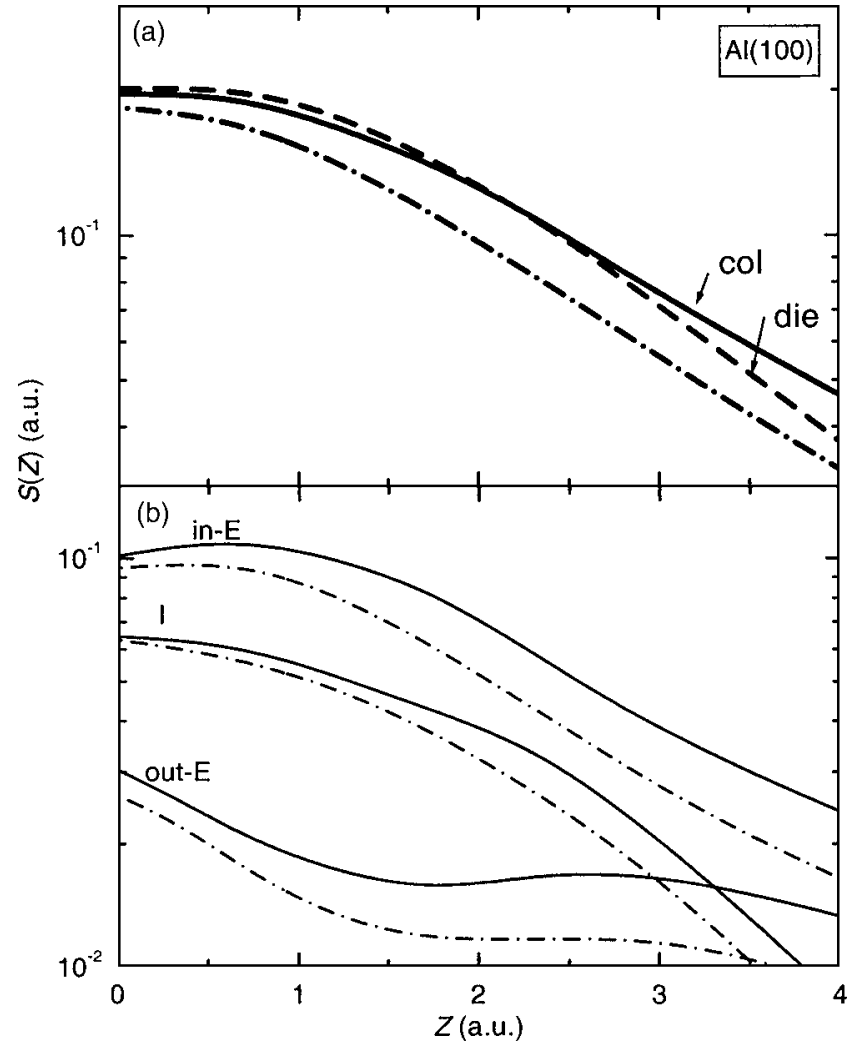

FIG. 3. Similar to Fig. 1 for $\mathrm{Al}(100)$ surfaces. Dash-dotted line, collisional BSB approximation without including the surface state. (a) Total energy loss per unit path. (b) Partial contributions from in$E$, out- $E$, and $I$ processes.

lence emission per unit path $d P(Z) / d \mathbf{k}_{f}$ is derived from Eq. (6) by defining the final electron momentum as $\mathbf{k}_{f}$ $=\left(\mathbf{k}_{f s}, k_{f z}\right)$, with $k_{f z}=\sqrt{2 \varepsilon_{n_{f}}}$, as in Ref. [17]. In the BSB model, for each positive energy $\varepsilon_{n_{f}}$ two functions $\phi_{n_{f}}$ are associated: the symmetric and the antisymmetric one (classified according to symmetry properties with respect to a plane parallel to the surface and placed in the middle of the slab). Then, the wave functions given by Eq. (4) do not allow us to distinguish the internal ionization process, associated with electrons emitted inside the solid, from the external ionization process, which corresponds to the emission of electrons toward the vacuum semispace. As a first estimation, in previous calculations [17] we considered that electrons ejected to the vacuum region were about $50 \%$ of the total ionized valence electrons. Here we have replaced this assumption by defining the final wave function associated with emission to the vacuum as a lineal combination of the symmetric and antisymmetric $\phi_{n_{f}}$ states. The coefficients of this transformation are obtained by matching the final wave function with the one corresponding to the external ionization process for the jellium surface potential [Eq. (A4) of Ref. [8]], for an arbitrary position in the vacuum side, far from the surface. The total emission probability along the whole trajectory is obtained by integrating $d P(Z) / d \mathbf{k}_{f}$ on the classical ion path. It has been calculated by representing the $P-S$ interaction with the Molière potential [20] plus the dynamic image potential given in Ref. [21].

Figure 4 shows the differential emission probability $d P / d \mathbf{k}_{f}$ as a function of the electron energy, for the incidence angle $\theta_{i}=1^{\circ}$. Collisional BSB probabilities for three electron observation angles $\theta_{e}=20^{\circ}, 45^{\circ}$, and $90^{\circ}$, measured with respect to the surface in the scattering plane, are displayed in the figure. Even though the collisional BSB method is found to overestimate low-energy excitations (see Fig. 2), this effect should not affect electron emission spectra for final energies $E_{f} \geqslant 10 \mathrm{eV}$. In Fig. 4 emission probabilities derived from the collisional BSB approximation are compared with values obtained from the same theory but without considering the surface state. We observe that the presence of the surface state introduces small differences for the angles $\theta_{e}$ $=45^{\circ}$ and $90^{\circ}$, while for $\theta_{e}=20^{\circ}$ the contribution of the surface state is negligible. Thus, for the considered collisional system the effect of the surface band structure on emission spectra seems to be of a lesser order, and it can only be appreciated in excitation processes.

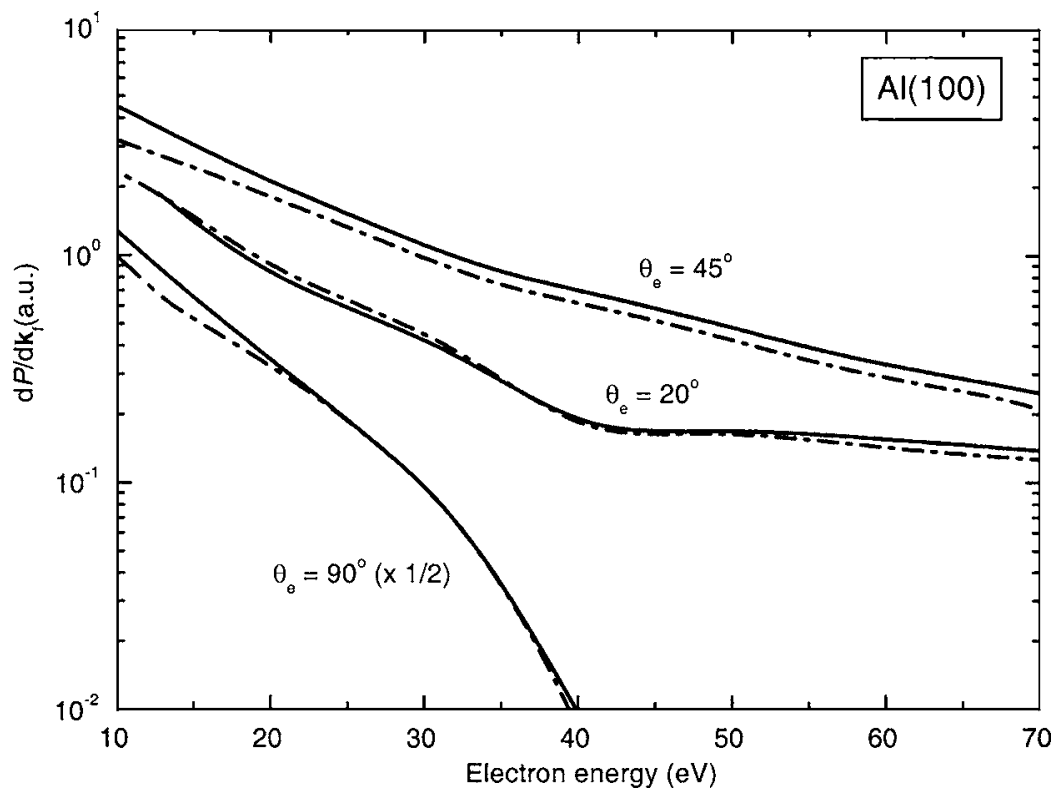

FIG. 4. Electron emission probability from the valence band for $100 \mathrm{keV}$ protons impinging on $\mathrm{Al}(100)$ surfaces with the incidence angle $\theta_{i}$ $=1^{\circ}$. Three electron ejection angles $\theta_{e}=20^{\circ}, 45^{\circ}$, and $90^{\circ}$, in the scattering plane, are displayed. Collisional BSB results: solid line, including the surface state; dash-dotted line, without including the surface state. 


\section{CONCLUSIONS}

We have calculated the energy loss per unit path using the BSB model within two different formalisms: the collisional and dielectric theories. Both methods were applied to protons scattered by aluminum surfaces. Unlike previous findings, where simpler surface descriptions were considered, we found that collisional and dielectric distance-dependent stopping powers coincide quite well for different projectilesurface distances. This agreement can be associated with the proper description of the perpendicular momentum distribution given by the BSB induced potential. The absence of plasmon excitations in the collisional model is compensated by a higher probability for low-energy excitations. The BSB model provides values of the distance-dependent stopping power that are in good agreement with experimental results [18]. Moreover, the collisional BSB approximation allowed us to analyze the different contributions to the lost energy. The main contribution comes from excitation processes to final states with perpendicular energy lower than Fermi level, while the energy lost by the ionization mechanism is around $40 \%$ smaller.

Since the BSB model incorporates features of the surface band structure, we also studied its influence on the energy loss by considering the $\mathrm{Al}(100)$ surface. This crystal surface presents an initially occupied surface state, whose contribution was found to be important for excitation processes, especially at long distances from the surface. In the ionization process, the presence of the surface state plays a minor role, and only affects weakly emission spectra for electron observation angles $\theta_{e} \gtrsim 45^{\circ}$.

\section{ACKNOWLEDGMENTS}

M.N.F. and M.S.G. acknowledge financial support from the ANPCyT (Grant No. PICTR 2002-00122), CONICET (Grant No. PEI 6417-01), and UBACyT (Grant No. X259). M.A., J.I.J, and V.M.S. acknowledge partial support by the Basque Departamento de Educación, Universidades e Investigación, the University of the Basque Country UPV/EHU (Grant No. 9/UPV 00206.215-13639/2001), the Spanish MCyT (Grants No. BFM2001-0076 and No. FIS2004-06490CO3-00), and the European FP6 Network of Excellence [Grant No. FP6-NoE NANOQUANTA(500198-2)]. M.A. and V. M. S. acknowledge financial support by the Gipuzkoako Foru Aldundia.
[1] H. Winter, Phys. Rep. 367, 387 (2002).

[2] J. I. Juaristi, F. J. García de Abajo, and P. M. Echenique, Phys. Rev. B 53, 13839 (1996).

[3] M. A. Cazalilla and F. J. García de Abajo, Nucl. Instrum. Methods Phys. Res. B 125, 106 (1997).

[4] M. A. Cazalilla and J. I. Juaristi, Nucl. Instrum. Methods Phys. Res. B 157, 104 (1999).

[5] J. E. Miraglia and M. S. Gravielle, Phys. Rev. A 66, 032901 (2002).

[6] R. Nuñez, P. M. Echenique, and R. H. Ritchie, J. Phys. C 13, 4229 (1980).

[7] A. García-Lekue and J. M. Pitarke, Phys. Rev. B 64, 035423 (2001).

[8] M. S. Gravielle, Phys. Rev. A 58, 4622 (1998).

[9] M. S. Gravielle, D. G. Arbó, and J. E. Miraglia, Nucl. Instrum. Methods Phys. Res. B 182, 29 (2002).

[10] M. S. Gravielle and J. E. Miraglia, Phys. Rev. A 65, 022901 (2002).

[11] E. V. Chulkov, V. M. Silkin, and P. M. Echenique, Surf. Sci.
391, L1217 (1997); 437, 330 (1999).

[12] M. Alducin, V. M. Silkin, J. I. Juaristi, and E. V. Chulkov, Phys. Rev. A 67, 032903 (2003).

[13] E. V. Chulkov, I. Sarría, V. M. Silkin, J. M. Pitarke, and P. M. Echenique, Phys. Rev. Lett. 80, 4947 (1998).

[14] J. Kliewer, R. Berndt, E. V. Chulkov, V. M. Silkin, P. M. Echenique, and S. Crampin, Science 288, 1399 (2000).

[15] T. Hecht, H. Winter, A. G. Borisov, J. P. Gauyacq, and A. K. Kazansky, Phys. Rev. Lett. 84, 2517 (2000).

[16] P. M. Echenique, R. Berndt, E. V. Chulkov, Th. Fauster, A. Goldmann, and U. Höfer, Surf. Sci. Rep. 52, 219 (2004).

[17] M. N. Faraggi, M. S. Gravielle, and V. M. Silkin, Phys. Rev. A 69, 042901 (2004).

[18] H. Winter, M. Wilke, and M. Bergomaz, Nucl. Instrum. Methods Phys. Res. B 125, 124 (1997).

[19] D. G. Arbó and J. E. Miraglia, Phys. Rev. A 58, 2970 (1998).

[20] V. G. Molière, Z. Naturforsch. A 2, 133 (1947).

[21] N. R. Arista, Phys. Rev. A 49, 1885 (1994). 